Elizabeth Costa Dias ${ }^{1}$

Thais Lacerda e Silva²

\section{Contribuições da Atenção Primária em Saúde para a implementação da Política Nacional de Saúde e Segurança no Trabalho (PNSST)}

\author{
Contributions of the Primary Health Care system for \\ implementation of the Brazilian National Occupational \\ Safety and Health Plan (PNSST)
}

\begin{abstract}
${ }^{1}$ Departamento de Medicina Preventiva e Social da Faculdade de Medicina da Universidade Federal de Minas Gerais (UFMG), Belo Horizonte, MG, Brasil.

${ }^{2}$ Escola Nacional de Saúde Pública (ENSP), Fundação Oswaldo Cruz (Fiocruz), Rio de Janeiro, RJ, Brasil.

Contato:

Elizabeth Costa Dias

E-mail:

bethdias@medicina.ufmg.br

Apoio Financeiro: Fundo

Nacional de Saúde (Projeto FNS

$\mathrm{n}^{\mathrm{o}}$ 18720.9380001/07-023).

As autoras declaram que este artigo não apresenta conflito de interesses.
\end{abstract}

Recebido: 02/03/2012

Revisado: 11/03/2013

Aprovado: 15/03/2013

\section{Resumo}

Introdução: o texto descreve a contribuição do Sistema Único de Saúde (SUS) para a atenção integral à saúde dos trabalhadores de acordo com a Política Nacional de Segurança e Saúde no Trabalho, considerando a organização das Redes de Atenção e o papel de coordenação atribuído à Atenção Primária em Saúde (APS). Método: realizaram-se revisão bibliográfica e análise de documentos normativos para identificar ações de atenção aos trabalhadores a serem desenvolvidas pelas equipes da APS. Resultados: as ações foram organizadas em seis grupos: a) diagnóstico situacional, incluindo a identificação das atividades produtivas; b) caracterização do perfil demográfico e epidemiológico dos trabalhadores; c) planejamento das ações com priorização de intervenções sobre problemas de saúde de maior frequência, risco e vulnerabilidade; d) assistência ao trabalhador vítima de acidente ou doença relacionada ao trabalho; e) ações educativas e de promoção da saúde; f) ações caracterizadas como de Vigilância em Saúde do Trabalhador. Discute-se as potencialidades e limitações da APS para desenvolver as ações e recomenda-se o fomento da educação permanente e o matriciamento das ações pelos Centros de Referência em Saúde do Trabalhador (Cerest) e outras instâncias do SUS.

Palavras-chave: política pública de saúde; saúde do trabalhador; atenção primária em saúde; política de segurança e saúde no trabalho.

\begin{abstract}
Introduction: This paper describes the contribution of the Brazilian Unified Health System (SUS) for a comprehensive approach to workers' health care as established by the National Occupational Safety and Health Plan (PNSST). It takes into account the Health Care Network organization, as well as the health surveillance coordination assigned to the Primary Health Care (PHC). Method: Through literature review and analysis of normative documents the authors identified health surveillance actions to be implemented by the PHC teams and their potentialities for workers' health purposes. Results: The identified actions were organized and discussed according to: a) a situational diagnosis, including work activities held in the region; b) workers' demographic and epidemiological profiles; c) planning of actions with priority to interventions aimed at the most frequent, hazardous and vulnerable health problems; d) assistance to victims of work-related injuries or diseases; e) educational actions and health promotion; f) workers' health surveillance actions. The authors discuss the PHC's potentialities and limitations in implementing Workers' Health Care actions and recommend that Workers' Health Reference Centers (Cerest) and other organizations included in the SUS system should provide permanent educational programs and appropriate technical support.
\end{abstract}

Keywords: public health policy; workers' health; primary health care; Brazilian national occupational safety and health plan (PNSST). 


\section{Introdução}

A recente publicação da Política Nacional de Segurança e Saúde no Trabalho (PNSST), pelo Decreto Presidencial $\mathrm{n}^{\mathrm{o}} 7.602$, de 07 de novembro de 2011 (BRASIL, 2011a), representa um marco histórico na abordagem das relações trabalho-saúde e doença no Brasil. Pela primeira vez, um documento oficial explicita as responsabilidades e ações a serem desenvolvidas pelos organismos de governo responsáveis pela proteção e recuperação da saúde dos trabalhadores. Busca-se, dessa forma, superar a fragmentação e a superposição das ações desenvolvidas pelos setores Trabalho, Previdência Social, Saúde e Meio Ambiente por meio da articulação das ações de governo, com participação voluntária das organizações representativas de trabalhadores e empregadores (BRASIL, 2011a).

A PNSST tem como princípios norteadores a universalidade, a integralidade, o diálogo social e a precedência das ações de promoção, proteção e prevenção sobre as de assistência e reabilitação. Os objetivos propostos são a promoção da saúde e a melhoria da qualidade de vida do trabalhador e a prevenção de acidentes e danos à saúde relacionados ao trabalho ou que ocorram no curso dele (BRASIL, 2011a).

A diretriz de incluir, no sistema nacional de promoção e proteção da saúde no trabalho, todos os trabalhadores brasileiros (os formalmente registrados de acordo com as prescrições da Consolidação das Leis do Trabalho, os autônomos, os domésticos e os informais, além de civis e militares, servidores da União, dos Estados e dos Municípios) é inovadora. Esse desafio é ampliado no contexto de transformações observadas no mundo do trabalho e no modelo prevalente de desenvolvimento adotado para o país, marcado pela precarização do trabalho e o desemprego estrutural, que reforçam a desigualdade social (DRUCK, 2011).

Assim, a efetiva implementação da PNSST exige políticas afirmativas de inclusão social e depende da participação dos trabalhadores, como destaca Facchini (2006) ao analisar a versão preliminar do documento apresentado como subsídio às discussões na $3^{\underline{a}}$ Conferência Nacional de Saúde do Trabalhador, realizada em 2005, sob a coordenação compartilhada dos Ministérios da Saúde, do Trabalho e Emprego e da Previdência Social.

O Plano Nacional de Segurança e Saúde no Trabalho, publicado em abril de 2012, detalha os aspectos operacionais da Política visando facilitar sua implementação (BRASIL, 2012a).

Entre as competências atribuídas ao Ministério da Saúde, o artigo VII, letra a, explicita o fomento da:
[...] atenção integral à saúde dos trabalhadores, envolvendo a promoção de ambientes e processos de trabalho saudáveis, o fortalecimento da vigilância de ambientes, processos e agravos relacionados ao trabalho, a assistência integral à saúde dos trabalhadores, incluindo a reabilitação física e psicossocial. (BRASIL, 2011a)

Entre os aspectos facilitadores para que o Ministério da Saúde desempenhe bem essa atribuição, o princípio constitucional da universalidade de acesso ao cuidado da saúde, presente também como um dos pilares para a organização e o funcionamento do Sistema Único de Saúde (SUS), antecipa e contempla a prescrição do Decreto $\mathrm{n}^{-}$7.602/11. Diferentemente da atuação do Ministério do Trabalho e Emprego e da Previdência Social, cujas práticas estão historicamente vinculadas aos trabalhadores do setor formal de trabalho, o SUS nasce sob a orientação de cuidar de todos os trabalhadores (BRASIL, 1990). A capilaridade da rede de serviços do SUS, presente em todos os municípios brasileiros, permite alcançar os trabalhadores, o mais próximo de onde moram e trabalham, por meio dos serviços da Atenção Primária à Saúde (APS).

Este texto discute a contribuição do SUS para a estruturação da atenção integral à saúde dos trabalhadores prescrita na PNSST, considerando o modelo da Rede da Atenção à Saúde e o papel ordenador e coordenador do cuidado atribuído à APS. No texto, as expressões "Atenção Básica" e "Atenção Primária à Saúde" são utilizadas como sinônimas, seguindo as orientações emanadas da Política Nacional de Atenção Básica de 2011 (BRASIL, 2011b).

\section{Métodos}

Inicialmente, a partir de pesquisa qualitativa, de cunho bibliográfico e análise de documentos normativos que orientam a organização das ações de Saúde do Trabalhador no SUS, foram identificadas as ações prescritas e/ou desenvolvidas no âmbito da APS consideradas no escopo da atenção integral à saúde dos trabalhadores. No que tange aos documentos normativos, destacam-se a Portaria $\mathrm{n}^{\mathrm{o}}$ 1.823/2012, que institui a Política Nacional de Saúde do Trabalhador e da Trabalhadora (BRASIL, 2012b); a Portaria no 2.488, de 2011 (BRASIL, 2011b), que explicita a Política Nacional de Atenção Básica; a Portaria $\mathrm{n}^{\circ} 4.279$ de 2010, que estabelece diretrizes para a organização da Rede de Atenção à Saúde (RAS) no âmbito do SUS (BRASIL, 2010); a Portaria $\mathrm{n}^{\mathrm{o}} 3.252$ de 2009 , que aprova as diretrizes para execução e financiamento das ações de Vigilância em Saúde (BRASIL, 2009a); e a Portaria no 2.728 , também de 2009 (BRASIL, 2009b), que redefiniu a organização e o funcionamento da Rede Nacional de Atenção Integral à Saúde do Trabalhador (Renast). 
Também foram incluídos na análise os instrumentos utilizados pelas equipes da APS, entre eles a ficha de cadastramento das famílias (Ficha A), a ficha de acompanhamento de grupos específicos e o registro de atividades, procedimentos e notificações (Ficha D) (BRASIL, 2000), buscando os campos referentes à Saúde do Trabalhador ou de interesse à ela.

As ações identificadas foram analisadas e cotejadas com os resultados do estudo "Desenvolvimento de conceitos e instrumentos facilitadores da inserção de ações de Saúde do Trabalhador na Atenção Primária à Saúde, com ênfase na Saúde da Família”3, desenvolvido por pesquisadores da área Saúde \& Trabalho da Faculdade de Medicina da Universidade Federal de Minas Gerais (UFMG), por encomenda da Coordenação Geral de Saúde do Trabalhador (CGSAT) do Departamento de Saúde Ambiental e Saúde do Trabalhador (DSAST) da Secretaria de Vigilância em Saúde (SVS) do Ministério da Saúde (SVS), com apoio do Fundo Nacional de Saúde (DIAS; LACERDA E SILVA, 2012).

Este estudo foi aprovado pelo Comitê de Ética em Pesquisa da Universidade Federal de Minas Gerais sob o parecer de número 256/08.

\section{Atenção integral à saúde do trabalhador no SUS}

O conceito de atenção integral à saúde do trabalhador é polissêmico. A Constituição Federal de 1988 define o atendimento integral como diretriz e reforça a prioridade das atividades preventivas, sem prejuízo dos serviços assistenciais (BRASIL, 1988). Porém, a Lei Orgânica $\mathrm{n}^{\circ} 8.080$ estabelece como princípio a integralidade da assistência, abrangendo as ações necessárias, em cada caso, em todos os níveis de atenção e, desse modo, permitindo a interpretação de que o termo se refere a ações assistenciais resolutivas (BRASIL, 1990).

De acordo com a PNSST, as ações de promoção e proteção da saúde e a prevenção de agravos e do adoecimento são indissociáveis da assistência, incluindo a reabilitação em nível individual e coletivo. Ao considerar os aspectos biológicos, sociopolíticos e culturais determinantes do processo saúde-doença, este conceito é o que mais se aproxima daquele proposto pelo movimento da Saúde do Trabalhador, o qual surge em sintonia com o processo de reorganização social e política do país ocorrido nos anos
1970-1980, enfatizando a contribuição do trabalho enquanto determinante das condições de vida e saúde. Além da atenção integral à saúde, propunha a ruptura com as práticas tradicionais da Saúde Ocupacional, a incorporação dos trabalhadores como sujeitos da própria saúde e a responsabilidade da rede pública de serviços de saúde no cuidado dos trabalhadores (MENDES; DIAS, 1991).

O desenvolvimento de ações de Saúde do Trabalhador na rede pública de serviços de saúde no Brasil, que consideram as relações produção-consumo, ambiente e saúde como determinantes do processo saúde-doença dos trabalhadores, é um processo sociopolítico e técnico que se inicia no final dos anos 1970 e foi institucionalizado pela Constituição Federal de 1988 e regulamentado pela Lei Orgânica da Saúde em 1990 (BRASIL, 1988; BRASIL, 1990).

Desde então, as ações de Saúde do Trabalhador no SUS têm sido desenvolvidas em distintas estratégias e formas de organização institucional nos três níveis de gestão do SUS. A criação da Renast, em 2002, representou um marco importante nesse processo, tendo os Centros de Referência em Saúde do Trabalhador (Cerest) como lócus privilegiado de execução, articulação e pactuação de ações de saúde, intra e intersetorialmente, ampliando a visibilidade da área de Saúde do Trabalhador junto aos gestores e ao controle social (DIAS; HOEFEL, 2005).

Em 2006, o Pacto pela Saúde redefiniu a organização da atenção à saúde no país e atribuiu à APS o papel de eixo organizador das ações no SUS (BRASIL, 2006). Posteriormente, a Portaria n⿳⺈ 4.279/2010 estabeleceu diretrizes para a organização da RAS no SUS, atribuindo à APS a função de centro de comunicação da rede (BRASIL, 2010).

O Ministério da Saúde vem desde 1988 buscando formular um documento que explicite a política de atenção à saúde dos trabalhadores no âmbito do SUS (PNST-SUS). A versão mais recente, construída sob a coordenação da área técnica de Saúde do Trabalhador do Ministério da Saúde, a CGSAT, foi aprovada pelo Conselho Nacional de Saúde em 2011. A PNST-SUS reitera a indissociabilidade das práticas preventivas e curativas, a necessidade, no âmbito do SUS, de uma ação transversal de caráter transdisciplinar e interinstitucional, a necessidade de sintonia e de uma relação dinâmica com as mudanças nos processos produtivos e a participação dos trabalhadores enquanto sujeitos das ações de saúde. De acordo com o documento, as estratégias definidas

\footnotetext{
${ }^{3}$ A produção técnica resultante desse estudo está disponibilizada no "Painel de Informações em Saúde Ambiental e Saúde do Trabalhador", do Ministério da Saúde e na Renast online e inclui três dissertações de Mestrado, dois trabalhos de conclusão de curso de especialização (TCC), três relatórios técnicos, cartilha e guia de apoio à capacitação de Agentes Comunitários de Saúde (ACS) e um relatório final.
} 
para a implantação da atenção integral à saúde do trabalhador compreendem: a) a integração da Vigilância em Saúde do Trabalhador (Visat) com os demais componentes da Vigilância em Saúde e com a APS; b) a análise do perfil produtivo e da situação de saúde dos trabalhadores; c) a estruturação da Rede de Atenção Integral à Saúde do Trabalhador; d) o fortalecimento e a ampliação da articulação intersetorial; e) o estímulo à participação da comunidade, dos trabalhadores e do controle social; f) o desenvolvimento e a capacitação de recursos humanos; g) o apoio ao desenvolvimento de estudos e pesquisas (BRASIL, 2012b).

A contribuição do SUS para a atenção integral à saúde do trabalhador cresce em importância no contexto do processo de reestruturação produtiva e das mudanças na natureza e nas relações do trabalho, que redefinem o perfil dos trabalhadores e do processo saúde-doença, acarretando necessidades e desafios que a sociedade e o aparelho estatal encontram dificuldades para responder (NEHMY; DIAS, 2010).

Entre as características dos processos produtivos contemporâneos, o crescimento do trabalho informal e domiciliado e o aumento da vulnerabilidade, da desproteção social e da degradação ambiental podem ser encontrados mesmo nas cadeias produtivas mais complexas de setores da economia considerados mais fortes e/ou tradicionais, como, por exemplo, a indústria metalúrgica, do vestuário, calçadista, de alimentos, entre outras. Etapas ou parcelas do processo de trabalho são desenvolvidas em pequenas unidades, por vezes no próprio domicílio do trabalhador. Geralmente são atividades que agregam menos valor e/ou consideradas mais "sujas", arriscadas e perigosas para a saúde (ANTUNES, 2004; NEHMY; DIAS, 2010).

Observa-se que o trabalho desenvolvido por microempresas ou no domicílio torna-se praticamente invisível, permanecendo à margem da regulação trabalhista, da inspeção, da fiscalização e da ação dos sindicatos e das organizações de trabalhadores (VIEIRA, 2009).

O modelo de desenvolvimento adotado no país aproxima os campos da Saúde do Trabalhador e da Saúde Ambiental pela compreensão de que os mesmos processos produtivos são responsáveis pela degradação do ambiente, em particular dos ambientes de trabalho, acarretando danos e agravos à saúde da população geral e dos trabalhadores (RIGOTTO, 2003).

Essa ampliação da percepção e da discussão acerca dos efeitos dos processos de produção e consumo presentes na sociedade moderna sobre a saúde humana e o ambiente tem sido acompanhada de crescente institucionalização das ações de Vigilância em
Saúde do Trabalhador e Saúde Ambiental nos três níveis de gestão do SUS, expressa na presença formal na estrutura organizacional e nos documentos normativos do atual modelo de atenção à saúde no país (NETTO et al., 2006; DIAS; LACERDA E SILVA; ALMEIDA, 2012).

Nesse cenário, para que o SUS seja capaz de prover atenção integral à saúde dos trabalhadores, é essencial que cada ponto de atenção do SUS e os setores responsáveis pela Vigilância em Saúde incorporem de forma sistemática a contribuição do trabalho enquanto determinante do processo saúde-doença das pessoas e da qualidade ambiental. A operacionalização da atenção integral depende da articulação entre diversos saberes, práticas e responsabilidades, da atuação inter e transdisciplinar e de sólida articulação intra e intersetorial.

A estratégia de organizar o SUS no modelo da RAS visa superar a fragmentação da atenção e gestão nas regiões de saúde e assegurar, aos usuários do SUS, ações e serviços necessários à resolução de seus problemas e necessidades de saúde, sendo definida como arranjos organizativos de ações e serviços de saúde, de diferentes densidades tecnológicas, que, integradas por meio de sistemas de apoio técnico, logístico e de gestão, buscam garantir a integralidade do cuidado (BRASIL, 2010; MENDES, 2010).

Nessa perspectiva, a Renast, cuja organização é anterior à Portaria no 4.279/2010 (BRASIL, 2010), vem buscando se adequar ao novo modelo, particularmente no que se refere às atribuições dos Cerest, que passam a assumir a tarefa de matriciar ou subsidiar tecnicamente a rede SUS nas ações de promoção, prevenção, vigilância, diagnóstico, tratamento e reabilitação em saúde dos trabalhadores urbanos e rurais. O fortalecimento da Visat e sua integração com os demais componentes da Vigilância em Saúde e com a APS são as principais diretrizes da PNST-SUS.

\section{Contribuição da APS para a atenção integral à saúde dos trabalhadores}

Entre as características da APS que justificam sua centralidade no modelo da RAS destacam-se: a presença em todos os 5.564 municípios brasileiros e a potencialidade de organizar ações e serviços de saúde com base nas necessidades e nos problemas de saúde da população; oferecer atenção contínua e integral por equipe multidisciplinar e por considerar o usuário-sujeito em sua singularidade e inserção sociocultural (BRASIL, 2010; BRASIL, 2011b). A APS deve ter capacidade resolutiva sobre os problemas mais comuns de saúde e é considerada como pri- 
meiro nível de atenção, a partir do qual se realiza e coordena o cuidado em todos os outros pontos de atenção (MENDES, 2010).

A atenção integral à saúde dos trabalhadores pode se beneficiar dessas características da APS, que favorecem: a oferta de serviços de saúde nos municípios, facilitando o acesso dos usuários-trabalhadores e a identificação de demandas e problemas de saúde relacionados às atividades produtivas desenvolvidas no território; o estabelecimento de vínculos e o fortalecimento de relações de confiança entre a equipe de saúde e a população, mediados pelo Agente Comunitário de Saúde; as ações de caráter multidisciplinar; a geração de informações de saúde mais fidedignas; e o estímulo à participação dos trabalhadores que estão organizados em formas menos tradicionais, que não os sindicatos (BRASIL, 2009c; SANTOS; RIGOTTO, 2011; LACERDA E SILVA; DIAS; SILVA, 2012).

O desenvolvimento de ações de saúde do trabalhador na APS possui amplo aparato legal e normativo, entre eles o Decreto no 7.508 de 28 de junho de 2011 (BRASIL, 2011c), que regulamenta a Lei Orgânica de Saúde (BRASIL, 1990), a PNST-SUS (BRASIL, 2012b), a Portaria $\mathrm{n}^{\circ}$ 4.279/2010 (BRASIL, 2010), a Portaria $\mathrm{n}^{\mathrm{0}} 3.252 / 2009$ (BRASIL, 2009a), que aprova as diretrizes para a organização da Vigilância em Saúde, e a Portaria no 2.728/2009 (BRASIL, 2009b), que dispõe sobre a organização da Renast. É importante que, no processo de organização das ações de saúde do trabalhador no âmbito do SUS, a centralidade da APS seja observada nesses documentos e sejam identificados os caminhos e as diretrizes apontados para a consolidação da atenção integral à saúde do trabalhador.

O documento da PNST-SUS orienta que as equipes da APS, de forma articulada com as demais instâncias da rede e com o apoio das áreas técnicas de saúde do trabalhador e dos Centros de Referência em Saúde do Trabalhador (BRASIL, 2012b), devem desenvolver ações no âmbito individual e coletivo, abrangendo a promoção e a proteção da saúde dos trabalhadores, a prevenção de agravos relacionados ao trabalho, o diagnóstico, o tratamento, a reabilitação e a manutenção da saúde.

O mesmo documento prescreve que o planejamento e o desenvolvimento das ações de saúde do trabalhador devem contemplar as especificidades dos perfis das atividades produtivas e da população trabalhadora, considerando os problemas de saúde deles advindos e sua distribuição nos territórios, cabendo à APS:

[...] considerar que os territórios são espaços sócio-políticos dinâmicos, com trabalhadores residentes e não residentes, executando atividades produtivas e de trabalho em locais públicos e privados, peri e intra-domiciliares. (BRASIL, 2012b, p. 19)
É importante considerar que a adscrição das famílias à Estratégia Saúde da Família (ESF) considera apenas o critério domiciliar, no território. Para a Saúde do Trabalhador, esta lógica necessita ser mudada, de modo a permitir o acesso aos serviços de saúde de trabalhadores que não residem, mas trabalham no local e ali passam tempo significativo de suas vidas. A equipe deve se responsabilizar pelos usuários trabalhadores que residem e os que trabalham no território de abrangência da unidade.

A proposta de prover atenção aos trabalhadores que não residem, mas trabalham no território vem sendo defendida também no âmbito da APS. No estudo de avaliação da implementação da ESF em dez grandes centros urbanos, desenvolvido pelo Ministério da Saúde em 2005, essa questão foi ressaltada principalmente em cidades "dormitórios" das regiões metropolitanas e o relatório recomenda a adscrição por local de trabalho ou por meio de inscrição individual dos usuários-trabalhadores em Unidades de Saúde da Família (USF) próximas aos locais de trabalho (ESCOREL, 2005).

A utilização do critério de territorialização para a organização do processo de trabalho e o planejamento das ações de saúde da APS também enseja a operacionalização de práticas de Vigilância em Saúde no âmbito da APS, na medida em que a equipe de saúde consegue compreender o território em sua riqueza e complexidade e seus contextos de uso (MONKEN; BARCELLOS, 2005).

Com frequência, os fatores de risco e perigos gerados pelos processos produtivos extrapolam os limites dos ambientes de trabalho e atingem as comunidades moradoras no entorno das unidades de produção e mesmo locais mais distantes. Neste contexto, as ações de vigilância em saúde do trabalhador devem ser inseridas no planejamento da atuação das equipes com base no conhecimento do perfil produtivo e dos fatores de riscos para a saúde da população, além do perfil dos trabalhadores. Para isso, é fundamental o apoio técnico e pedagógico dos setores da Vigilância em Saúde e do Cerest às equipes. Nesse sentido, a Portaria no 3.252/2009 estabelece que a integração entre a Vigilância em Saúde e a APS é diretriz obrigatória para a construção da integralidade do cuidado (BRASIL, 2009a).

Outra contribuição importante para o cuidado integral aos usuários trabalhadores são as ações desenvolvidas pelos ACS. Em suas práticas cotidianas de trabalho, eles reconhecem, por vezes de modo intuitivo, a contribuição do trabalho no processo saúde-doença das pessoas. Além disso, conhecem os processos produtivos instalados no território e estabelecem relações entre essas atividades e o perfil ocupacional das famílias e a saúde das pessoas, suas 
queixas, demandas e problemas que possam estar relacionadas ao trabalho. Porém, os ACS se queixam da falta de continuidade do cuidado aos trabalhadores, ressentem-se da falta de preparo técnico para entender e orientar sobre essas questões e das dificuldades de interlocução com as equipes, que muitas vezes não valorizam suas observações (LACERDA E SILVA; DIAS; SILVA, 2012).

Embora se reconheça as possibilidades e a relevância das contribuições da APS para a atenção integral à saúde, é importante considerar as dificuldades estruturais que perpassam a efetivação do papel da APS no SUS, na atualidade. A desvalorização social traduzida na ausência de um plano de carreira gera alta rotatividade dos profissionais, particularmente dos médicos. Também são significativos: a deficiência da infraestrutura, refletida na precariedade da rede física; a inadequação das condições de trabalho; o baixo índice de conectividade e informatização; a ausência de fluxos bem definidos e eficientes de regulação; a hegemonia das ações assistenciais, que ocupam a maior parte do tempo de trabalho das equipes; deficiências na formação e de suporte técnico para o enfrentamento de situações e o desenvolvimento de ações de alta complexidade; e a sobrecarga de trabalho das equipes, entre outras.

\section{Lições aprendidas: o que as práticas das equipes da APS estão nos ensinando?}

As práticas das equipes de saúde da APS, envolvendo ações de cuidado diferenciado aos usuários-trabalhadores, considerando sua inserção nos processos produtivos, estão registradas na literatura técnica sob a forma de dissertações de mestrado, artigos em periódicos e, de modo expressivo, nos relatos de experiências em congressos e reuniões técnicas da área da Atenção Básica e da Saúde Coletiva (DIAS; LACERDA E SILVA, 2012).

De modo esquemático, essas ações podem ser organizadas em seis grandes eixos: a) diagnóstico situacional, que inclui o levantamento e/ou a identificação das atividades produtivas desenvolvidas em um dado território; b) caracterização do perfil demográfico dos usuários-trabalhadores que residem no território e o perfil epidemiológico traduzido nos agravos e nas formas de adoecimento prevalentes; c) o planejamento das ações, com a priorização de intervenções sobre os problemas de saúde de maior frequência, risco e vulnerabilidade; d) definição dos procedimentos de assistência ao trabalhador vítima de acidente ou doença relacionada ao trabalho, organizados em linhas de cuidado; e) ações educativas e de promoção da saúde, com orientação sobre fatores de risco para a saúde e medidas de proteção e direitos previdenciários; e f) ações caracterizadas como de Visat (DIAS, LACERDA E SILVA, 2012).

\section{O diagnóstico situacional do território}

A implantação do trabalho de uma equipe da APS em um dado território é sempre precedida da elaboração de um diagnóstico local de situação. Assim, quando se propõe contemplar as questões de Saúde do Trabalhador é necessário incorporar a este diagnóstico inicial duas informações básicas: que atividades produtivas são desenvolvidas nesse território e quem são os trabalhadores que ali residem (e, idealmente, ali trabalham). E, se possível, de que adoecem e morrem os trabalhadores que compõem a população adscrita no território de uma equipe de saúde.

Na rotina de trabalho das equipes, esse reconhecimento já é feito, principalmente, pelos ACS no processo de realização do "Mapeamento do Território" ou "Diagnóstico Local" conforme denominado em algumas regiões. Esse diagnóstico, em geral, é feito por ocasião da implantação da Unidade Básica de Saúde em um determinado território, mas deve ser atualizado periodicamente. Ele é a base para que a equipe conheça seu território de atuação e a população sob sua responsabilidade. O conhecimento do território pela equipe de saúde e a percepção deles sobre questões-problemas relacionadas ao trabalho foram destacados no estudo de Dias, Bertolini e Pimenta (2011).

É importante conhecer o perfil dos usuários-trabalhadores, considerando, minimamente: a idade, o sexo, a escolaridade e a ocupação; a identificação de chefes de família desempregados e em outra situação de vulnerabilidade social e possíveis doenças ou agravos que podem estar associadas. A identificação dos processos produtivos instalados no território permite inferir alguns dos fatores de risco e perigos para a saúde e o ambiente associados às atividades produtivas. Essas informações coletadas, organizadas e discutidas pela equipe de saúde estão sendo denominadas, neste texto, como Diagnóstico em Saúde do Trabalhador.

A identificação dos usuários-trabalhadores e suas características, como sexo, idade e ocupação, podem ser obtidas na Ficha A, de cadastramento familiar. É atribuição do ACS cadastrar todas as famílias adscritas às unidades básicas de saúde, sendo coletadas e registradas na ficha diversas informações sobre essas famílias (LACERDA E SILVA; DIAS; RIBEIRO, 2011).

Já a identificação dos processos produtivos instalados no território é realizada no momento do Mapeamento da Área de Atuação da equipe de saúde, em que os ACS identificam no mapa hospitais, escolas, igrejas, postos policiais, campo de futebol, quadra de esporte, rios, locais propensos a inundações, depósitos onde 
são despejados lixos, fábricas, comércio em geral, entre outros. O mapeamento dos processos produtivos é essencial para o planejamento e a execução das ações de saúde pelas equipes, considerando que as atividades produtivas interferem na condição de vida e saúde das pessoas. Esse mapeamento permite inferir o perfil de adoecimento, bem como os possíveis riscos e perigos para a saúde dos trabalhadores, da população e também para o ambiente, advindos dessas atividades.

O Mapeamento da Área de Atuação permite ao ACS e aos outros membros da equipe: conhecer os caminhos mais fáceis para chegar aos locais necessários; identificar as barreiras geográficas que dificultam o caminho das pessoas até os serviços de saúde; conhecer a realidade da comunidade e planejar como resolver os problemas de saúde com mais eficácia; planejar as visitas domiciliares; identificar as microáreas de risco; entre outras questões. O mapa consiste em uma ferramenta indispensável para o trabalho da equipe de saúde, devendo representar o desenho de todo o território de atuação (BRASIL, 2009a).

O preenchimento adequado do campo "doenças ou condições referidas”, presente na Ficha A, também permite a aproximação quanto ao perfil epidemiológico e de possíveis agravos e doenças relacionadas ao trabalho. Em geral, os ACS preenchem neste campo as condições e/ou doenças referidas pelos membros das famílias, tais como diabetes, hipertensão, tuberculose, entre outras. Além dessas condições, é importante que o agente seja orientado a preencher o item acidente de trabalho (AT) nos casos em que o usuário referir o ocorrido ou mesmo doença relacionada ao trabalho (DRT).

Na visita para cadastramento das famílias, o ACS também deve ser orientado sobre a importância do preenchimento do campo "Observações", com vistas à identificação da existência de atividades de trabalho desenvolvidas nos espaços domiciliares e peridomiciliares, como, por exemplo, costura, produção e venda de alimentos, manicure, produção de produtos de limpeza, entre outros.

\section{O planejamento da atuação da equipe e as ações de assistência}

O planejamento das ações direcionadas aos trabalhadores, a serem desenvolvidas pela equipe de saúde, deve se basear nas informações sistematizadas nas etapas do Diagnóstico Situacional, no cadastramento das famílias e em informações sobre o perfil epidemiológico dos trabalhadores. A partir delas é possível priorizar as intervenções sobre os problemas de saúde dos trabalhadores considerando a frequência, a gravidade e a vulnerabilidade.
Outra fonte importante de informação para o planejamento das ações são as queixas dos trabalhadores que procuram a UBS na demanda espontânea. Para qualquer ação de cuidado a esse usuário-trabalhador é essencial que o profissional de saúde que o acolha investigue se suas queixas ou problemas de saúde podem estar relacionadas ao trabalho que realiza.

Nesse aspecto, é importante lembrar que os trabalhadores podem adoecer ou morrer por causas não relacionadas ao trabalho, mas frequentemente o trabalho ou as condições em que este é realizado são responsáveis diretamente pelo agravo ou adoecimento, ou contribuem para torná-lo mais precoce, mais grave ou de tratamento mais difícil. Este conjunto conforma o elenco das doenças relacionadas ao trabalho, que no Brasil compõem a Lista Brasileira de Doenças Relacionadas ao Trabalho (BRASIL, 1999).

O reconhecimento do usuário enquanto trabalhador começa no preenchimento da Ficha A, conforme já assinalado, por meio do registro da ocupação dos membros da família. Embora seja um instrumento importante, sua utilização para subsidiar a suspeita da relação entre o adoecimento e o trabalho é frágil, pois os ACS não são preparados para este registro, além da ficha não contemplar a ocupação pregressa e sim a atual. Outras abordagens feitas pelas equipes da APS e que constituem momentos importantes de se reconhecer o usuário enquanto trabalhador e coletar informações sobre sua história ocupacional são o acolhimento e a consulta médica ou do enfermeiro.

Tradicionalmente, na formação médica e dos enfermeiros, pouca ou nenhuma atenção é dada à coleta da história ocupacional, o que faz com que os profissionais, em geral, tenham dificuldades em inseri-la em suas práticas de trabalho (CHIAVEGATTO, 2010). Entre as perguntas básicas que devem ser feitas ao trabalhador estão: o que faz; como faz; que produtos e instrumentos utiliza; em que condições executa o trabalho; há quanto tempo; em que ritmo; quais as exigências de produtividade, entre outras (DIAS; LAUAR, 2012).

Ouvir o trabalhador falando de seu trabalho, de suas impressões e sentimentos, de como seu corpo reage no trabalho e fora dele é a tradução prática da recomendação feita aos médicos por Ramazzini quanto à necessidade de perguntar a seus pacientes: “Qual é a sua profissão?” e considerar a resposta no raciocínio clínico (RAMAZZINI, 2000).

A escuta especializada do trabalhador, valorizada na atualidade na qualificação e humanização do cuidado, permite conhecer as tarefas mais frequentes que ele executa, as exigências em termos de esforço físico, posturas, gestos e movimentos, a descrição de produ- 
tos usados com respectivas quantidades e tempo de uso, a presença ou não de cheiros e/ou interferências em atividades (por exemplo, ruído e comunicação), o número de peças produzidas, a intensidade e as formas de controle de ritmos de trabalho, as interações existentes com outras tarefas, os imprevistos e os incidentes que podem aumentar as exposições, os dados do ambiente físico, como tipo de instalação, layout, contaminação por contiguidade, ruído, emanações, produtos intermediários, ventilação, medidas de proteção coletivas e individuais (DIAS; LAUAR, 2012).

Em alguns casos, a investigação de uma doença relacionada ao trabalho, identificada a partir da anamnese clínico-ocupacional, pode demandar informações complementares a serem buscadas, por exemplo, na bibliografia especializada ou junto à empresa onde o paciente trabalha ou trabalhou.

Ao término da coleta da história, deve ser feito um resumo no prontuário contendo, no mínimo: as funções desempenhadas pelo trabalhador com o respectivo tempo de trabalho e os principais fatores de risco a que o paciente esteve exposto, um breve inventário dos riscos ocupacionais e a possível relação com as queixas ou os achados clínicos. Este procedimento facilitará o raciocínio clínico, o estabelecimento de nexo entre o trabalho e a doença apresentada pelo paciente e servirá como orientação para os procedimentos propedêuticos, terapêuticos e demais condutas a serem adotadas.

A coleta da história ocupacional é essencial para: auxiliar no diagnóstico e na definição do plano terapêutico e estabelecer a relação entre o agravo ou doença e o trabalho; possibilitar a notificação do agravo no Sistema de Informação de Agravo de Notificação (Sinan), quando pertinente; identificar possíveis riscos e perigos envolvidos no trabalho do usuário; orientar o trabalhador sobre seus direitos trabalhistas e previdenciários e sobre a gênese, a evolução e a prevenção de sua patologia; e desencadear ações de vigilância e intervenções em ambientes de trabalho de modo articulado com a Vigilância em Saúde.

Considerando que os problemas de saúde relacionados ao trabalho muitas vezes são complexos e não podem ser resolvidos no âmbito da APS, para que esta coordene, de fato, o cuidado integral da saúde do usuário-trabalhador é essencial contar com o apoio dos demais pontos de atenção à saúde, de modo a garantir o diagnóstico correto e o estabelecimento da relação do adoecimento com o trabalho a fim de definir adequadamente o plano terapêutico. Para isso, é necessária a estruturação do fluxo de referência e contrarreferência do usuário-trabalhador, e contar com fluxos e processos de regulação estabelecidos em linhas de cuidado, construídas para cada um dos agravos prevalentes, orientadas pelas infor- mações obtidas no diagnóstico situacional e perfil das atividades produtivas presentes no território.

\section{Ações educativas, de promoção da saúde e orientação dos usuários-trabalhadores}

O desenvolvimento de ações educativas e de promoção da saúde integra, conceitualmente, o cotidiano das equipes da APS e são realizadas no domicílio, na UBS e em outros espaços comunitários. Para a Saúde do Trabalhador, essas ações têm o propósito de ampliar a compreeensão sobre o papel do trabalho na produção do processo saúde-doença e esclarecer e identificar os fatores de risco para a saúde presentes no trabalho, as medidas de prevenção e controle, as obrigações dos empregadores e os direitos assegurados ao trabalhador pelas políticas públicas. Elas devem contribuir para o empoderamento dos trabalhadores na luta por melhores condições de vida e de trabalho.

As ações educativas requerem o estabelecimento de uma relação de confiança com o usuário, o vínculo que possibilita o diálogo e exige dos ACS e dos demais profissionais o aperfeiçoamento da escuta do usuário sobre suas condições de vida e saúde, sua percepção sobre o trabalho, bem como sobre os fatores de riscos presentes na atividade, as repercussões sobre a saúde e as medidas de proteção disponíveis.

São exemplos de ações educativas a serem desenvolvidas com o usuário-trabalhador pela equipe da APS, com destaque para os ACS: a) orientação dos trabalhadores sobre a presença de perigos para saúde na atividade e as medidas de proteção e prevenção de acidentes e doenças relacionadas ao trabalho; b) informações sobre os serviços de saúde disponíveis, os fluxos ou caminhos na rede de cuidado à sua saúde; os fluxos de atendimento e o acompanhamento do usuário-trabalhador em qualquer ponto de atenção em que ele esteja; c) orientações sobre os direitos trabalhistas e previdenciários (LACERDA E SILVA; DIAS; RIBEIRO, 2011).

Muitas das atividades educativas podem e devem ser desenvolvidas em grupo, aproveitando aqueles já formados na unidade, como, por exemplo, os grupos de hipertensos e de gestantes, para discutir aspectos das relações trabalho-saúde. Também podem ser organizados novos grupos considerando os interesses: grupo etário, agravos e situações de vulnerabilidade ou exposição.

O ACS desempenha papel fundamental no desenvolvimento das ações educativas, atuando como mediador, tradutor e elo entre as equipes de saúde e a comunidade. Como membro da equipe e morador do território em que trabalha, possui amplo conhe- 
cimento sobre os processos produtivos instalados no território e a influência desses na saúde das pessoas e também sobre o ambiente. Conhece de modo particular o perfil ocupacional das famílias e as principais queixas, demandas e problemas que possam estar relacionadas ao trabalho. Porém, para desenvolver bem seu trabalho, ele necessita ser capacitado e contar com suporte técnico permanente.

\section{A Vigilância da Saúde do Trabalhador na APS}

A Vigilância em Saúde é, na atualidade, um dos grandes desafios para o SUS e para a APS em particular. Conceitualmente, a Vigilância em Saúde envolve ações de promoção, proteção, prevenção e controle das doenças e agravos à saúde e abrange os seguintes componentes: Vigilância Epidemiológica, Sanitária, Vigilância em Saúde Ambiental, Visat, Vigilância da Situação de Saúde e Promoção da Saúde (BRASIL, 2009a).

A Visat compreende: um conjunto de ações e práticas de vigilância dos agravos relacionados ao trabalho; intervenções sobre fatores de risco, ambientes e processos de trabalho; o acompanhamento de indicadores para avaliação da situação de saúde; e articulação de ações de promoção da saúde (BRASIL, 2012b).

Para que essas ações sejam efetivadas na APS, é essencial que os setores de Vigilância Epidemiológica, Sanitária e Ambiental, além de outros níveis técnicos do SUS, apoiem as equipes, desenvolvendo ações compartilhadas e garantindo o fluxo e a resolutividade das ações, como, por exemplo: a discussão de casos considerados mais "complexos", a elaboração conjunta de roteiros de inspeção, de vistorias em ambientes de trabalho, a análise da situação de saúde, entre outras.

Nos fóruns de discussão da Saúde do Trabalhador, como no X Congresso da Abrasco ${ }^{4}$, realizado em Porto Alegre, tem sido questionada a adequação de se denominar ações desenvolvidas pelas equipes da APS como "de vigilância em saúde do trabalhador", uma vez que não cumprem alguns dos pressupostos básicos da Visat, entre eles o envolvimento dos trabalhadores em todas as etapas do processo e o foco na transformação das condições de trabalho geradoras de doença. Entretanto, é importante reconhecer que, apesar de incompletas, muitas dessas ações representam práticas inovadoras das equipes e possibilitam identificar situações-problema, muitas vezes complexas e que desencadeiam ações em articulação com as Vigilâncias Epidemiológica, Sanitária e Ambiental e de cunho in- terinstitucional, em parceria com a fiscalização do Ministério do Trabalho e Emprego.

A experiência e os estudos disponíveis têm mostrado que as equipes da APS consideram os fatores determinantes da saúde presentes no meio ambiente e atuam sobre eles, e essa experiência pode ser estendida aos ambientes de trabalho. Porém, as intervenções sobre os determinantes com frequência requerem atuação integrada com setores de Meio Ambiente, do Trabalho, Ministério Público, entre outros (REDE NACIONAL DE ATENÇÃO INTEGRAL À SAÚDE DO TRABALHADOR, 2011; VILAS BOAS; DIAS, 2008; FERNANDES, 2012).

Outra ação importante de vigilância refere-se à investigação e à notificação ao Sinan de agravos à saúde relacionados ao trabalho, prescrita na Portaria $\mathrm{n}^{\circ}$ 104/2011. Nesse sentido, é muito importante reverter o atual quadro de sub-registro e dar visibilidade ao problema para que as questões de saúde do trabalhador entrem na agenda técnica e política dos gestores e do controle social do SUS. Alguns fatores que contribuem para a subnotificação podem ser facilmente corrigidos, como, por exemplo, a exigência de que apenas sejam notificados casos confirmados, com exceção das intoxicações exógenas, e de que as notificações sejam realizadas pelos serviços da rede sentinela da Renast.

A Ficha D, utilizada pelas equipes da APS para registrar atividades, procedimentos e notificação é fonte de informação importante para a análise de situação de saúde dos trabalhadores. Nela, devem ser registrados mensalmente os atendimentos específicos de acidentados no trabalho. Embora esse registro seja importante, é preciso modificar a orientação disponível no Sistema de Informação da Atenção Básica (Siab) para o preenchimento do campo, o qual define que deverão ser registrados apenas acidentes de trabalho para o qual foi emitida a Comunicação de Acidente de Trabalho (CAT), que é um instrumento da Previdência Social e se refere apenas aos acidentes ocorridos com trabalhadores segurados pelo Seguro de Acidente de Trabalho (SAT) e fere o princípio da universalidade do SUS.

Outro instrumento importante na coleta de informações sobre as condições de vida e saúde da população é a Ficha A. No campo de doenças e/ou condições referidas, recomenda-se que os ACS incluam as siglas AT (acidente de trabalho) e DRT (doença relacionada ao trabalho), devendo ser previamente preparados para reconhecer usuários-trabalhadores com agravos relacionados ao trabalho. Esses registros devem ser acompanhados e analisados pelas equipes com o apoio de técnicos das vigilâncias. 
Sintetizando, são exemplos de ações de Visat que devem ser incorporadas ao processo de trabalho das equipes da APS: a) mapeamento dos processos produtivos do território e identificação dos riscos e perigos à saúde associados às atividades; b) identificação do perfil ocupacional e de trabalhadores mais vulneráveis (desempregados, trabalho infantil, trabalho domiciliar, entre outros); c) notificação no Sinan de agravos relacionados ao trabalho e acompanhamento da situação de saúde dos trabalhadores para definição de ações prioritárias; d) articulação intrassetorial para a vigilância dos processos e ambientes de trabalho; e e) promoção da saúde, com ênfase no empoderamento dos trabalhadores e orientações sobre a prevenção de riscos e perigos (BRASIL, 2011d; DIAS; LACERDA E SILVA; ALMEIDA, 2012).

Nas ações de vigilância de ambientes de trabalho domiciliar, busca-se conhecer as condições presentes no ambiente de trabalho, visando corrigir, diminuir ou eliminar as situações perigosas para a saúde dos trabalhadores e familiares, por vezes idosos e crianças, mais vulneráveis. A colaboração e a participação dos trabalhadores é essencial tanto para identificação e reconhecimento dos riscos que envolvem o desenvolvimento de seu trabalho, quanto para a definição das medidas a serem adotadas para proteger a saúde.

Sintetizando, as ações de Visat na APS são: a) suporte técnico para a investigação dos agravos de saúde do trabalhador de notificação compulsória; b) apoio à construção, mapeamento e análise do perfil produtivo e do perfil de morbimortalidade da população trabalhadora; c) vigilância de ambientes de trabalho de forma integrada com a Vigilância em Saúde e outros setores.

As ações de vigilância no âmbito da APS, em especial a vigilância dos ambientes de trabalho, deve contar com o apoio de outros dispositivos do SUS, como a Vigilância Sanitária, a Visat, a Vigilância Ambiental e os Cerest. Muitos municípios, principalmente nos de menor porte, possuem apenas os setores de Vigilância Epidemiológica e Vigilância Sanitária. Nesses casos, recomenda-se a ampliação do objeto de intervenção dessas vigilâncias, incluindo as condições de trabalho e de saúde dos trabalhadores.

\section{Desafios e proposições para o desenvolvi- mento de ações de Saúde do Trabalhador}

Assim, ao se pensar em desenvolver ou fomentar ações de Saúde do Trabalhador na APS são necessárias mudanças nos processos de trabalho das equipes e sólido investimento na educação permanente e no suporte técnico das ações. Outra questão a ser enfrentada é o fortalecimento dos mecanismos de participação dos trabalhadores, particularmente do setor informal de trabalho.

Finalizando essas reflexões sobre a importância e as possibilidades das ações de Saúde do Trabalhador na APS, pode-se dizer que essas são possíveis e já existem, apesar de assistemáticas e descontínuas. Para inserir as ações de vigilância nas práticas cotidianas das equipes de saúde de Atenção Básica/ Saúde da Família, os profissionais necessitam desenvolver habilidades, utilizar as ferramentas de investigação, registro, análise, programação e planejamento de modo a organizar ações previstas e de atenção à demanda espontânea a fim de mudar a qualidade de vida das pessoas do território onde atuam.

Entre as dificuldades a serem superadas para sua ampliação e real institucionalização nas práticas cotidianas das equipes, a mais urgente a ser equacionada se refere à sobrecarga de trabalho das equipes submetidas a exigências de produzir um conjunto de ações prescritas, às quais devem responder para garantir o cumprimento das metas estabelecidas (CHIAVEGATTO, 2010; DIAS, 2010).

Neste sentido, a consolidação do cuidado integral à saúde dos trabalhadores na APS requer algumas ações, entre elas a incorporação, no cotidiano das equipes, do conceito de que os processos produtivos desenvolvidos no território e as suas relações com o ambiente e a saúde das pessoas determinam necessidades de saúde que devem ser abordadas por meio da articulação de ações intra e intersetoriais, de processos sólidos de educação permanente, além da adoção da metodologia de apoio matricial das ações, conforme previsto na Portaria no 3.252/09 (BRASIL, 2009a).

Outra questão importante refere-se à modificação do processo de trabalho das equipes, que até o momento enfatiza o desenvolvimento de ações assistenciais e o cumprimento de metas pré-estabelecidas. Embora, esteja previsto que a equipe trabalhe com base nas necessidades locorregionais, identificadas a partir do Diagnóstico Situacional, observa-se que muitas ainda estão centradas no desenvolvimento dos programas prioritários do governo federal, a exemplo do Programa Saúde da Criança. Além disso, é necessário que o ACS seja inserido de fato na equipe, devendo ser valorizadas e utilizadas no planejamento das ações de saúde, as informações coletadas por ele junto à comunidade.

A educação permanente deve possibilitar a qualificação das práticas de cuidado, gestão e participação popular. Para a Saúde do Trabalhador, a qualificação dessas práticas pode se dar pela inclusão da temática nos cursos pré-formatados e pela estruturação de processos de educação permanente que partem da vivência das equipes de saúde e dos problemas identificados que relacionam o processo trabalho-saúde-doença. 
Finalmente, é importante pensar no apoio matricial do trabalho das equipes da APS. Conceitualmente, o apoio matricial consiste em arranjo organizacional e uma metodologia de trabalho que visa oferecer suporte técnico e pedagógico em áreas específicas (DIMENSTEIN et al., 2009). A vinculação da estratégia de apoio matricial aos processos de educação permanente potencializa em muito o desenvolvimento de conhecimento e habilidades de gestão do cui- dado aos usuários-trabalhadores pela APS. O apoio matricial às equipes da APS para o desenvolvimento de ações de saúde do trabalhador pode ser visto, na atualidade, como desafio e oportunidade para a redefinição do papel dos Cerest. Porém, além dos Cerest, são potenciais matriciadoras dessas ações, as referências técnicas estaduais e municipais em Saúde do Trabalhador e profissionais da Vigilância em Saúde, em especial da Vigilância em Saúde do Trabalhador.

\section{Contribuições de autoria}

Ambas autoras participaram no delineamento, na análise e na elaboração do manuscrito.

\section{Referências}

ANTUNES, R. As mutações no mundo do trabalho na era da mundialização do Capital. Educação e Sociedade, Campinas, v. 25, n. 87, p. 335-351, 2004.

BRASIL. Constituição (1988). Diário Oficial [da] República Federativa do Brasil, Brasília, DF, 05 out. 1988. Disponível em: <http://www.planalto.gov.br/ ccivil_03/constituicao/constituicaocompilado.htm >. Acesso em: 24 maio 2013.

Presidência da República. Casa Civil. Lei $\overline{\mathrm{n}^{\circ} \mathrm{8}}$ 8.080, de 19 de setembro de 1990. Dispõe sobre as condições para a promoção, proteção e recuperação da saúde, a organização e o funcionamento dos serviços correspondentes e dá outras providências. Diário Oficial [da] República Federativa do Brasil, Brasília, DF, 20 set. 1990. Disponível em: <http://www.planalto.gov.br/ ccivil_03/leis/l8080.htm>. Acesso em: 13 maio 2013.

. Ministério da Saúde. Portaria nº 1.339, de 18 de novembro de 1999. Lista de doenças relacionadas ao trabalho. Diário Oficial [da] República Federativa do Brasil, Brasília, DF, 19 nov. de 1999. Disponível em: <http://dtr2001.saude.gov.br/sas/PORTARIAS/Port99/ GM/GM-1339.html>. Acesso em: 10 abr. 2013.

SIAB. Manual do Sistema de Informação da Atenção Básica. Brasília: Ministério da Saúde, 2000.

. Portaria nº 399/GM, de 22 de

fevereiro de 2006. Divulga o Pacto pela Saúde 2006 - Consolidação do SUS e aprova as Diretrizes Operacionais do Referido Pacto. Disponível em: <http://dtr2001.saude.gov.br/sas/PORTARIAS/ Port2006/GM/GM-399.htm>. Acesso em: 20 mar. 2013.

. Portaria no $3.252 / G M$, de 22 de

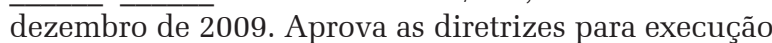
e financiamento das ações de vigilância em saúde pela União, estados, Distrito Federal e municípios e dá outras providências. 2009a. Disponível em: <http:// www.brasilsus.com.br/legislacoes/gm/102068-3252>. Acesso em: 20 março 2013.
. Portaria $\mathrm{n}^{\mathrm{o}} 2.728$, de 11 de novembro de 2009. Dispõe sobre a Rede Nacional de Atenção Integral à Saúde do Trabalhador (Renast) e dá outras providências. Diário Oficial [da] República Federativa do Brasil, Brasília, DF, 12 nov. 2009b. Disponível em: <http://portal.saude.gov.br/portal/arquivos/pdf/portaria renast_2728.pdf >. Acesso em: 20 março 2013.

. Secretaria de Atenção à Saúde.

Departamento de Atenção Básica. O Trabalho do Agente Comunitário de Saúde. Brasília: Ministério da Saúde, 2009c.

. Portaria $\mathrm{n}^{\mathrm{o}} 4.279$, de 30 de dezembro de 2010 . Estabelece diretrizes para a organização da Rede de Atenção à Saúde no âmbito do Sistema Único de Saúde (SUS). Disponível em: <http://www. brasilsus.com.br/legislacoes/gm/107038-4279.html>. Acesso em: 13 maio 2013.

. Presidência da República. Casa Civil. Decreto n⿳⺈ 7.602, de 07 de novembro de 2011. Dispõe sobre a Política Nacional de Segurança e Saúde no Trabalho - PNSST. Diário Oficial [da] República Federativa do Brasil, Brasília, DF, 8 nov. 2011a. Disponível em: <http://www.planalto.gov.br/ ccivil_03/_Ato2011-2014/2011/Decreto/D7602.htm>. Acesso em: 13 maio 2013.

. Ministério da Saúde. Portaria nº 2.488/

GM, de 21 de outubro de 2011. Aprova a Política Nacional de Atenção Básica, estabelecendo a revisão de diretrizes e normas para a organização da Atenção Básica, para a Estratégia Saúde da Família (ESF) e o Programa de Agentes Comunitários de Saúde (PACS). Diário Oficial [da] República Federativa do Brasil, Brasília, DF, 24 out. 2011b. Disponível em: <http:// www.brasilsus.com.br/legislacoes/gm/110154-2488. html>. Acesso em: 13 maio 2013.

. Decreto n ${ }^{\circ} 7.508 / \mathrm{GM}$, de 28 de junho de 2011. Regulamenta a Lei no 8.080, de 19 de setembro de 1990, para dispor sobre a organização 
do Sistema Único de Saúde - SUS, o planejamento da saúde, a assistência à saúde e a articulação interfederativa, e dá outras providências. Diário Oficial [da] República Federativa do Brasil, Brasília, DF, 28 jun. 2011c. Disponível em: < http://www.brasilsus. com.br/legislacoes/gm/108599-7508.html>. Acesso em: 13 maio 2013.

. Portaria $\mathrm{n}^{\mathrm{o}}$ 104/GM, de 25 de janeiro de 2011. Define as terminologias adotadas em legislação nacional, conforme o disposto no Regulamento Sanitário Internacional 2005 (RSI 2005), a relação de doenças, agravos e eventos em saúde pública de notificação compulsória em todo o território nacional e estabelece fluxo, critérios, responsabilidades e atribuições aos profissionais e serviços de saúde. Diário Oficial [da] República Federativa do Brasil, Brasília, DF, 26 jan. 2011d. Disponível em: < http://bvsms.saude.gov. br/bvs/saudelegis/gm/2011/prt0104_25_01_2011.html $>$. Acesso em: 13 maio 2013 .

. Comissão Tripartite de Saúde e Segurança no Trabalho. Plano Nacional de Segurança e Saúde no Trabalho. Brasília/DF, abril de 2012a.

. Portaria $\mathrm{n}^{\mathrm{o}}$ 1.823/GM, de 23 de agosto de 2012. Institui a Política Nacional de Saúde do Trabalhador e da Trabalhadora. Diário Oficial [da] República Federativa do Brasil, Brasília, DF, 24 ago. 2012b. Disponível em: <http://bvsms.saude.gov.br/ bvs/saudelegis/gm/2012/prt1823_23_08_2012.html >. Acesso em: 20 março 2013.

CHIAVEGATTO, C. V. Percepção dos profissionais de nível superior da atenção primária quanto ao desenvolvimento de ações de saúde do trabalhador no SUS em Minas Gerais. 2010. 128 f. Dissertação (Mestrado em Saúde Pública)-Faculdade de Medicina, Universidade Federal de Minas Gerais, Belo Horizonte, 2010.

DIAS, E. C. (Coord.). Desenvolvimento de ações de saúde do trabalhador na Atenção Primária à Saúde: aspectos históricos, conceituais, normativos e diretrizes. Belo Horizonte: UFMG, 2010. 63 p. (Relatório técnico-científico). Disponível em: <http://www.saude.rs.gov.br/ upload/1337000708_Desenvolvimento\%20de\%20 A\%С3\%A7\%C3\%B5-es\%20de\%20Sa\%C3\%BAde\%20 do\%20Trabalhador.pdf $>$. Acesso em: 8 mar. 2013.

DIAS, E. C; HOEFEL, M. G. O desafio de implementar as ações de saúde do trabalhador no SUS: a estratégia da Renast. Ciência e Saúde Coletiva, Rio de Janeiro, v. 10, n. 4, p. 817-828, 2005.

DIAS, E. C.; LACERDA E SILVA, T. Desenvolvimento de conceitos e instrumentos facilitadores da inserção de ações de Saúde do Trabalhador na Atenção Primária de Saúde, no SUS. Relatório Final (agosto 2008 a dezembro 2012). Belo Horizonte: UFMG, 2012.

DIAS, E. C.; LACERDA E SILVA, T.; ALMEIDA, M. H. C. Desafios para a construção cotidiana da vigilância em saúde ambiental e em saúde do trabalhador na Atenção Primária à Saúde. Cadernos de Saúde Coletiva, Rio de Janeiro, v. 20, n. 1, p. 15-24, 2012.

DIAS, E. C.; LAUAR, I. D. Doenças relacionadas com o trabalho: diagnóstico e ações decorrentes. In. PEDROSO, E. R. P.; ROCHA, M. O. C. (Org.). Clínica Médica. 2. ed. São Paulo: Atheneu, 2012. No prelo.

DIAS, M. D. A.; BERTOLINI, G. C. S.; PIMENTA, A. L. Saúde do trabalhador na Atenção Básica: análise a partir de uma experiência municipal. Trabalho, Educação e Saúde, Rio de Janeiro, v. 9, n. 1, p. 137 148, jun. 2011.

DIMENSTEIN, M. et al. O apoio matricial em USF: experimentando inovações em saúde mental. Saúde e Sociedade, São Paulo, v. 18, n. 1, p. 63-74, 2009.

DRUCK, G. Trabalho, precarização e resistências: novos e velhos desafios? Caderno CRH, Salvador, v. 42, n. 1, p. 35-55, 2011. Número especial.

ESCOREL, S. (Coord.). Saúde da Família: avaliação da implementação em dez grandes centros urbanos síntese dos principais resultados. 2. ed. atual. Brasília, DF: Ministério da Saúde, 2005.

FACCHINI, L. A. Vigilância em saúde do trabalhador: uma aproximação prática. Boletim da Saúde, Porto Alegre, v. 20, n. 1, p. 50-56, jan./jun. 2006.

FERNANDES, L. M. M. Ações de saúde do trabalhador na Atenção Primária de Saúde: estudo de caso de experiência desenvolvida no SUS de Minas Gerais. 87 f. 2012. Dissertação (Mestrado em Saúde Pública)-Faculdade de Medicina, Universidade Federal de Minas Gerais, Belo Horizonte, 2012.

LACERDA E SILVA, T., DIAS, E. C.; RIBEIRO, E. C. O. Saberes e práticas do Agente Comunitário de Saúde na atenção à Saúde do Trabalhador. Interface Comunicação, Saúde, Educação, São Paulo, v. 15, n. 38, p. 859-870, 2011.

LACERDA E SILVA, T.; DIAS, E. C.; SILVA, J. M. da. Contribuição do Agente Comunitário de Saúde na produção do cuidado aos trabalhadores. Revista Médica de Minas Gerais, 2012. (no prelo).

MENDES, E. V. As redes de atenção à saúde. Ciência $\mathcal{E}$ Saúde Coletiva, Rio de Janeiro, v. 15, n. 5, p. 2297-2305, ago. 2010.

MENDES, R.; DIAS, E. C. Da medicina do trabalho à saúde do trabalhador. Revista de Saúde Pública, São Paulo, v. 25, n. 5, p. 341-349, 1991.

MONKEN, M.; BARCELLOS, C. Vigilância em saúde e território utilizado: possibilidades teóricas e metodológicas. Cadernos de Saúde Pública, Rio de Janeiro, v. 21, n. 3, p. 898-906, maio-jun. 2005.

NEHMY, R. M. Q.; DIAS, E. C. Os caminhos da saúde do trabalhador: para onde apontam os sinais? Revista Médica de Minas Gerais, Minas Gerais, v. 20, n. 2, p. 13-23, 2010. Suplemento. 
NETTO, G. F. et al. Saúde e ambiente: reflexões para um novo ciclo do SUS. In: CASTRO, A.; MALO, M. SUS: ressignificando a promoção da saúde. São Paulo: Hucitec; OPAS, 2006. p. 152-170.

RAMAZZINI, B. As doenças dos trabalhadores. 3. ed. Tradução Raimundo Estrela. São Paulo: Fundacentro, 2000.

REDE NACIONAL DE ATENÇÃO INTEGRAL À SAÚDE DO TRABALHADOR. Desenvolvimento de ações de saúde do trabalhador na Atenção Primária à Saúde. Belo Horizonte, 2011.

Disponível em: <http://www.renastonline.org/ recursos/desenvolvimento-a $\% \mathrm{C} 3 \% \mathrm{~A} 7 \% \mathrm{C} 3 \% \mathrm{~B} 5 \mathrm{es}-$ sa\%C3\%BAde-trabalhador-aten\%C3\%A7\%C3\%A3oprim\%C3\%A1ria-sa\%C3\%BAde>. Acesso em: 8 mar. 2013.

RIGOTTO, R. M. Saúde ambiental e saúde dos trabalhadores: uma aproximação promissora entre o verde e o vermelho. Revista Brasileira de Epidemiologia, São Paulo, v. 6, n. 4, p. 388-404, 2003.

SANTOS, A. L., RIGOTTO, R. M. Território e territorialização: incorporando as relações produção, trabalho, ambiente e saúde na atenção básica à saúde. Trabalho Educação e Saúde, Rio de Janeiro, v. 8, n. 3, p. 387-406, 2011.

VIEIRA, M. C. F. O trabalho domiciliar e sua relação com a saúde do trabalhador: uma revisão da literatura brasileira no período de 2000 a 2009. 2009. $60 \mathrm{f}$. Dissertação. (Mestrado em Saúde Pública e Meio Ambiente)-Escola Nacional de Saúde Pública Sérgio Arouca, Fundação Oswaldo Cruz, Rio de Janeiro: 2009.

VILAS BOAS, S. W.; DIAS, E. C. Contribuição para a discussão sobre as políticas no setor sucro-alcooleiro e as repercussões sobre a saúde dos trabalhadores. In: PLATAFORMA BNDES. Impactos da indústria canavieira no Brasil. [Rio de Janeiro]: IBASE, 2008. (Versão Preliminar). 